\title{
Deviation from one-dimensionality in stationary properties and collisional dynamics of matter-wave solitons
}

\author{
Lev Khaykovich ${ }^{1}$ and Boris A. Malomed ${ }^{2}$ \\ ${ }^{1}$ Department of Physics, Bar-Ilan University, Ramat-Gan, 52900 Israel, and \\ ${ }^{2}$ Department of Interdisciplinary Studies, School of Electrical Engineering, \\ Faculty of Engineering, Tel Aviv University, Tel Aviv 69978, Israel
}

\begin{abstract}
By means of analytical and numerical methods, we study how the residual three-dimensionality affects dynamics of solitons in an attractive Bose-Einstein condensate loaded into a cigar-shaped trap. Based on an effective 1D Gross-Pitaevskii equation that includes an additional quintic selffocusing term, generated by the tight transverse confinement, we find a family of exact one-soliton solutions and demonstrate stability of the entire family, despite the possibility of collapse in the 1D equation with the quintic self-focusing nonlinearity. Simulating collisions between two solitons in the same setting, we find a critical velocity, $V_{c}$, below which merger of identical in-phase solitons is observed. Dependence of $V_{c}$ on the strength of the transverse confinement and number of atoms in the solitons is predicted by means of the perturbation theory and investigated in direct simulations. The simulations also demonstrate symmetry breaking in collisions of identical solitons with a nonzero phase difference. This effect is qualitatively explained by means of an analytical approximation.
\end{abstract}

\section{INTRODUCTION}

It is well known that a trapped atomic Bose-Einstein condensate (BEC) with attractive interactions is stable if the number of atoms in it is below a critical value, above which collapse occurs [1]. Beneath the collapse threshold, the BEC can form stable wave packets in a one-dimensional (1D) "cigar-shaped" trap, which is tightly confined in two (transverse) directions, and is unbound along the longitudinal axis. In that case, the stability of bright solitons is provided by balance between the quantum pressure, alias matter-wave (MW) dispersion, and mean-field attraction. A single MW soliton 22] and trains of interacting solitons [3] have been created in the cigar-shaped optical traps. However, while the trapping geometry was nearly one-dimensional, the solitons themselves were far from being 1D objects. In particular, in Ref. [2], a stable soliton was only possible if its longitudinal size exceeded the transverse size by no more than $20 \%$ (note that the situation was affected by an expulsive axial potential, unavoidable in the specific experimental setup). Recently, it was shown that the proximity of the soliton to being a 3D object strongly affects its properties, such as the character of its motion [4] and interactions [5, 6]. In particular, it was demonstrated that a moving soliton immersed in a cloud of thermal atoms is subjected to a temperature-dependent friction force [4]. A collision between two solitons, which are by themselves stable, in a confined geometry may readily lead to collapse, if the total number of atoms in the soliton pair exceeds the above-mentioned critical value, and the phase difference between the solitons is (close to) zero [6]. The significance of the effective dimensionality of MW solitary pulses is further emphasized by the recent observation of formation of a set of nearly-3D mutually repulsive MW solitons (with a phase shift of $\pi$ between them) as a result of incomplete collapse in an attractive BEC with the number of atoms several times larger than the critical value [5, 7].

In addition to being a profoundly important object of fundamental studies, MW solitary waves are also natural candidates for applications, such as high-precision atom interferometry and quantum-information processing. Thus, a thorough understanding of deviations of their behavior from that of ideal 1D solitons is important in this respect too.

In this paper we report results of theoretical investigation of the shape of stationary MW solitons and binary collisions between them in the quasi-1D regime, with the aim to identify manifestations of nonsolitonic behavior due to the residual multi-dimensionality. The effect of the tightly confined transverse dimensions is taken into account through a perturbative self-focusing quintic term added to the corresponding one-dimensional Gross-Pitaevskii equation (GPE), as per Refs. [8] and [4]. In Section II, we introduce this extended GPE, find a family of its exact one-soliton solutions, and demonstrate stability of the entire family, despite the fact that collapse occurs in the 1D equation with the quintic self-focusing term. In Section III, we investigate soliton collisions within the framework of this equation. On the contrary to completely elastic collisions between solitons in the cubic GPE (alias cubic nonlinear Schrödinger equation, NLSE), in the presence of the quintic term colliding solitons with zero phase difference, $\Delta \varphi=0$, merge into a single pulse if their relative velocity is smaller than a critical value, $2 V_{c}$. We find the dependence of $V_{c}$ on the strength of the transverse confinement and number of atoms in the solitons. For moderate quintic nonlinearity, good agreement with an analytic prediction derived from the perturbation theory is found. With a stronger quintic term, the numerical results deviate from the perturbation theory, although not dramatically. Finally, we demonstrate dynamical symmetry breaking between identical solitons colliding with $\triangle \varphi \neq 0$ (in that case, the merger does not 
occur), as a function of the relative velocity. An explanation to the latter effect is proposed. It is based on estimation of a symmetry-breaking parameter, which is a mismatch between the amplitude center and phase center of the soliton pair with $\Delta \varphi \neq 0$ (exact definitions are given below). Reasonably good agreement between numerical results and the analytical approximation is observed. The paper is concluded by Section IV.

\section{AN EFFECTIVE ONE-DIMENSIONAL GROSS-PITAEVSKII EQUATION AND EXACT SOLITON SOLUTIONS}

\section{A. Basic equations}

We start with the standard GPE for a condensate tightly confined in the transverse plane, with the radial coordinate $r$, and unconfined in the axial direction, $x$ :

$$
i \hbar \frac{\partial \psi}{\partial t}=-\frac{\hbar^{2}}{2 m}\left(\nabla_{\perp}^{2}+\frac{\partial^{2}}{\partial x^{2}}\right) \psi+\frac{1}{2} m \omega^{2} r^{2} \psi+\frac{4 \pi \hbar^{2} a}{m}|\psi|^{2} \psi
$$

where operator $\nabla_{\perp}^{2}$ acts in the transverse plane, $\omega$ is the frequency of the trapping potential in this plane, $m$ is the atomic mass, and $a<0$ is the scattering length. Transition to the quasi-1D description is possible if the change of the chemical potential due to the mean-field interaction is much smaller than the level spacing in the transverse trapping potential. We briefly recapitulate the corresponding derivation, following, chiefly, Ref. [4]. In the quasi-1D limit, the factorized ansatz, $\psi(r, x, t)=\phi(x, t) \chi(r, x, t)[9]$, is used to adiabatically separate fast transverse and slow longitudinal dynamics, by neglecting derivatives of $\chi$ with respect to the slow variables, $x$ and $t$. By substituting the ansatz into Eq. (1), two decoupled equations are obtained, within the framework of the tight-transverse-confinement approximation:

$$
\begin{gathered}
i \hbar \frac{\partial \phi}{\partial t}=-\frac{\hbar^{2}}{2 m} \frac{\partial^{2} \phi}{\partial^{2} x}+\tilde{\mu} \phi, \\
\tilde{\mu} \chi=-\frac{\hbar^{2}}{2 m} \nabla_{\perp}^{2} \chi+\frac{1}{2} m \omega^{2} r^{2} \chi+\frac{4 \pi \hbar^{2} a}{m} n|\chi|^{2} \chi,
\end{gathered}
$$

where the transverse chemical potential, $\tilde{\mu}$, has to be found from the ground-state solution of Eq. (3) as a function of the $1 \mathrm{D}$ density, $n(x, t) \equiv|\phi(x, t)|^{2}$. Physical solutions of Eq. (3) exist only if $-a n<0.47$ [10], otherwise transverse collapse occurs [1]. In the quasi-1D limit, corresponding to $-a n<<0.47$, the transverse wave function, $\chi$, is close to the ground state of the $2 \mathrm{D}$ harmonic potential, and can be expanded over the set of transverse eigenmodes, $\varphi_{m}(r)$ : $\chi(r, x)=\varphi_{0}(r)+\Sigma_{m} C_{m}(x) \varphi_{m}(r)$. Coefficients $C_{m}$ are small and can be calculated perturbatively. Accordingly, the transverse chemical potential $\tilde{\mu}$ can be expanded over powers of the density by means of the perturbative theory, $\tilde{\mu}=\hbar \omega+g_{1 \mathrm{D}} n-g_{2} n^{2}+\ldots$, where

$$
g_{1 \mathrm{D}}=2 \hbar \omega a, g_{2}=24\left(\ln \frac{4}{3}\right) \hbar \omega a^{2},
$$

as shown in Ref. [8] (the subscript "1D" implies that the corresponding coefficient appertains to the standard 1D model). Substituting the expansion for $\tilde{\mu}$ in Eq. (2), one arrives at an effective equation describing the soliton dynamics in the quasi-1D limit:

$$
i \hbar \frac{\partial \phi}{\partial t}=-\frac{\hbar^{2}}{2 m} \frac{\partial^{2} \phi}{\partial^{2} x}+g_{1 \mathrm{D}}|\phi|^{2} \phi-g_{2}|\phi|^{4} \phi,
$$

which is NLSE with the cubic-quintic (CQ) nonlinearity.

Other approaches to the derivation of the effective 1D GPE were also proposed [12, 14]. In particular, a more complex equation with nonpolynomial (algebraic) nonlinearity was derived, by means of the variational approach to the separation of the axial and transverse wave functions, in Ref. 12]. Expanding the nonlinearity up to the quintic term, one arrives at an equation similar to Eq. (5), but with a different numerical coefficient.

NLSEs with the CQ nonlinearity are well known as model equations in nonlinear optics, starting with pioneer works 16. GPEs with the CQ nonlinearity were also used in order to take into account three-body collisions in the BEC [17. However, in the previously considered settings, these equations were always considered with a combination of 
self-focusing cubic and self-defocusing quintic terms. A drastic difference in the present case is that the quintic term is self-focusing [as seen from Eq. (4), this conclusion does not depend on the sign of scattering length $a$, i.e., on the self-focusing or defocusing character of the cubic term; the same conclusion follows from the expansion of the above-mentioned nonpolynomial NLSE derived in Ref. [12]]. The use of the GPE with the "double-self-focusing" $\mathrm{CQ}$ nonlinearity, which is the case here, was tacitly assumed impossible, as in this case the equation gives rise to collapse. Nevertheless, we will show below that this equation generates meaningful stable solutions. In fact, if the cubic nonlinearity is self-focusing, i.e., the scattering length is negative (the case considered throughout the present work), the presence of the collapse is a relevant qualitative feature of the effective GPE, as collapse takes place too in the full 3D equation, from which Eq. (5) was derived (even if the strong collapse in the full 3D GPE and weak collapse in the 1D CQ equation bear essential differences). As shown in Refs. 9, 13, 15], the collapse in the 3D equation may be avoided under the constraint of $N|a| / a_{\perp}<0.627$, where $N$ is the number of atoms in the condensate, and $a_{\perp}=\sqrt{\hbar /(m \omega)}$ is the harmonic-oscillator length corresponding to the transverse confinement.

\section{B. Soliton solutions}

Below, we use Eq. (5) in the normalized form,

$$
i \frac{\partial \phi}{\partial t}=-\frac{1}{2} \frac{\partial^{2} \phi}{\partial^{2} x}+g_{1 \mathrm{D}}|\phi|^{2} \phi-g_{2}|\phi|^{4} \phi,
$$

where $g_{1 \mathrm{D}}<0$ and $g_{2}>0$ are dimensionless interaction constants. In fact, the absolute values of both of them may be additionally scaled to be 1 , but we find it more convenient to keep these coefficients as free parameters.

A family of exact soliton solutions to Eq. (6) can be found as an analytical continuation of the well-known solution of the equation with the self-defocusing quintic term [16]. The result is

$$
\begin{aligned}
\phi(x, t) & =2\left(\frac{3}{4 g_{2}}\right)^{1 / 4} e^{-i \mu t} \sqrt{\frac{-\mu}{\sqrt{g^{2}-4 \mu} \cosh (2 \sqrt{-2 \mu} x)+g}}, \\
g & \equiv-\frac{1}{2} \sqrt{\frac{3}{g_{2}}} g_{1 \mathrm{D}},
\end{aligned}
$$

where $\mu$ is the soliton's chemical potential that may take any value from $0<-\mu<\infty$. The squared amplitude of this soliton, i.e., the maximum atomic density at its center, is

$$
A^{2}=\frac{1}{2} \sqrt{\frac{3}{g_{2}}}\left(\sqrt{g^{2}-4 \mu}-g\right)
$$

and the norm of the soliton, which measures the total number of atoms, is

$$
N_{\mathrm{sol}} \equiv \int_{-\infty}^{+\infty}|\phi(x)|^{2} d x=\sqrt{\frac{6}{g_{2}}} \tan ^{-1}\left(\frac{2 \sqrt{-\mu}}{\sqrt{g^{2}-4 \mu}+g}\right) .
$$

The soliton's norm and squared amplitude are shown, as functions of the chemical potential, in Fig. [1

It is worth to note a drastic difference of this soliton family from its counterpart in the model with the self-defocusing quintic term, i.e., $g_{2}<0$ : in that case, the norm takes all values, $0<N_{\text {sol }}<\infty$, while the chemical potential is limited to a finite interval, $0<-\mu<|\mu|_{\max } \equiv 3 g_{1 \mathrm{D}}^{2} /\left(16\left|g_{2}\right|\right)$. On the contrary, for the present solution family, Eq. (10) demonstrates that the norm is limited to a finite interval,

$$
0<N_{\text {sol }}<N_{\max }=\sqrt{\frac{3}{8 g_{2}}} \pi,
$$

while $-\mu$ is not limited from above (as said above). In fact, $N_{\max }$ in Eq. (11) is a collapse border of the soliton family. Further, in the usual CQ model, with $g_{2}<0$, the amplitude is limited by a finite value, $A^{2}<3 g_{1 \mathrm{D}} /\left(4 g_{2}\right)$, while the width of the soliton diverges $\sim \ln \left(\left(|\mu|_{\max }-|\mu|\right)^{-1}\right)$ at $|\mu| \rightarrow|\mu|_{\max }$. In contrast to this, Eqs. (7) and (9) show that the amplitude of the present soliton family diverges, $A^{2} \approx \sqrt{-\left(3 / g_{2}\right) \mu}$, at $\mu \rightarrow-\infty$, and the width of the soliton shrinks in the same limit, as $1 / \sqrt{-\mu}$. This asymptotic behavior of the soliton solution clearly suggests a transition to a collapsing solution at $N_{\text {sol }} \rightarrow N_{\max }$, see Eq. (11). 

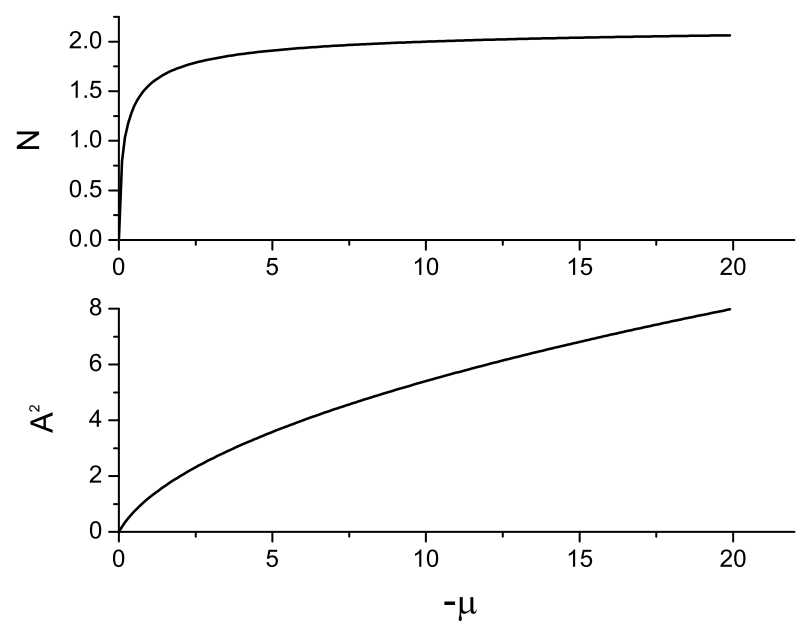

FIG. 1: The upper and lower panels display, respectively, the family of exact soliton solutions (7), with $g_{1 \mathrm{D}}=-1$ and $g_{2}=3 / 4$ [hence, $g=1$, see Eq. [8] ], in terms of the dependences of the norm and squared amplitude vs the chemical potential, as per Eqs. (10) and (9).

Equation (10) shows that condition $d N / d \mu<0$ holds for the entire soliton family (see also the upper panel in Fig. (1), hence the solitons satisfy the known Vakhitov-Kolokolov (VK) stability criterion [18]. As this criterion is only a necessary one, but not sufficient, the stability of the solitons was tested in systematic direct simulations of Eq. (6). Results clearly suggest that all the solitons are indeed stable against small perturbations (of course, a large perturbation may provoke onset of the collapse).

It is relevant to mention that the 1D GPE with the full algebraic nonlinearity introduced in Ref. 12 gives rise to two branches of (implicit) soliton solutions, one stable and one unstable; the branches meet and disappear at the point of transition to collapsing solutions. Equation (6) does not give rise to the second branch, as the combination of the cubic and quintic terms may be regarded as a truncated expansion of the full algebraic nonlinearity from the above-mentioned equation, and this truncation does not pick up the unstable branch.

It may also be relevant to note that, starting the derivation of the effective $1 \mathrm{D}$ equation from the $3 \mathrm{D}$ GPE with the positive scattering length (corresponding to self-repulsive BEC), one will arrive at Eq. (6) with $g_{1 \mathrm{D}}>0$ (and again with $g_{2}>0$ ). The corresponding equation, featuring competition between the cubic self-focusing and quintic self-defocusing terms, has a family of exact soliton solutions given by the same expressions (7)-(10), in which $g$ is negative, as per Eq. (8). Despite the formal similarity, the latter soliton family is completely different from the one presented above. In particular, in the limit of $\mu \rightarrow 0$ the solution is not a usual broad small-amplitude soliton, but rather an algebraic one,

$$
\phi_{\mu=0}(x)=\left(\frac{3}{g_{2}}\right)^{1 / 4} \sqrt{\frac{-g}{1+2 g^{2} x^{2}}} .
$$

The most drastic difference of the soliton family with $g_{1 \mathrm{D}}>0$ and $g_{2}>0$ from the above one is that it features $d N / d \mu>0$, hence this entire family is unstable, according to the VK criterion [algebraic solitons, such as one in Eq. (12), are known to be unstable for a different reason 19]]. Besides the fact that all the solitons in the model with the positive scattering length are unstable, their physical meaning is doubtful also because the quintic term, which appears as a perturbative correction to the cubic one [8], actually dominates over it in these solutions.

\section{SOLITON COLLISIONS}

\section{A. Merger of colliding solitons with $\Delta \varphi=0$}

It is commonly known that collisions between solitons in the one-dimensional NLSE, which is an integrable equation, are completely elastic. The force of interaction between the solitons depends on the relative phase between them: with $\triangle \varphi=0$ and $\Delta \varphi=\pi$, they are attract and repel each other, respectively [20, 21]. The quintic term breaks the 
integrability of the equation, and is expected to make collisions inelastic. For $\triangle \varphi=0$, simulations reveal a critical collision velocity, below which two identical solitons merge into a single one.

\section{Analytical considerations}

The merger may be explained by the fact that radiation loss due to the inelastic collision becomes greater than the initial kinetic energy of the soliton pair 21]. This explanation can be implemented in an explicit form if the quintic term is treated as a small perturbation. To this end, defining $\Psi \equiv \sqrt{\left|g_{1 \mathrm{D}}\right|} \phi$, we rewrite Eq. (6) in the following form:

$$
i \frac{\partial \Psi}{\partial t}=-\frac{1}{2} \frac{\partial^{2} \Psi}{\partial^{2} x}-|\Psi|^{2} \Psi-\epsilon|\Psi|^{4} \Psi
$$

where $\epsilon \equiv g_{2} / g_{1 \mathrm{D}}^{2}$. In the zero-order approximation $(\epsilon=0)$, the traveling-soliton solution to Eq. (13) is

$$
\Psi(x, t)=A \operatorname{sech}(A(x-V t)) e^{-i(\mu t-V x)},
$$

where $A$ and $V$ are its amplitude and velocity, and the frequency $\omega=V^{2} / 2+\mu$ is a sum of the kinetic energy and binding (potential) energy, $\mu=-A^{2} / 2$, per particle.

The use of the perturbation theory makes it possible to obtain the following analytical result for the collision between solitons with equal amplitudes $A$, velocities $\pm V$, and a phase shift $\triangle \varphi_{0}$ between them [21]: if the solitons are fast, $V^{2} \gg A^{2}$, the energy loss generated by the emission of radiation during the collision is

$$
(\triangle E)_{\mathrm{rad}}=\epsilon^{2} A^{2}\left\{\alpha A^{5}+V^{5} e^{-\pi V / A}\left[\beta_{1} \cos (\triangle \varphi)+\beta_{2} \sin (\triangle \varphi)\right]\right\}
$$

where $\alpha \approx 1381, \beta_{1} \approx 2401$ and $\beta_{2} \approx 347$. Note that the phase-dependent terms are exponentially small. In the same approximation, the collision-induced loss of the number of atoms is

$$
(\triangle N)_{\mathrm{rad}}=\left(2 / V^{2}\right)(\triangle E)_{\mathrm{rad}}
$$

To estimate a merger condition (threshold), we assume that the velocities $\pm V$, which determine the collision-induced losses as per Eqs. (15) and (16), are actually acquired by originally quiescent (or slowly moving) solitons due to their mutual attraction (if $\triangle \varphi$ is close to zero). To this purpose, we note that the effective potential of the interaction between far separated identical solitons is, in the case of $\epsilon=0$,

$$
U_{\text {int }}\left(X, \triangle \varphi_{0}\right)=-8 A^{3} e^{-A X} \cos \left(\triangle \varphi_{0}\right)
$$

[20], and the effective mass of the soliton is $M_{\text {eff }}=2 A$. In this approximation, the attraction accelerates the two inphase solitons to self-acquired velocities, $\pm V_{\text {self }}$, that can be found from the energy-balance equation, $2 \cdot\left(M_{\mathrm{eff}} V_{\text {self }}^{2} / 2\right)=$ $8 A^{3}$, hence $V_{\text {self }}=2 A$. Substituting this velocity in Eq. (15) shows that the phase-dependent part is less than $10 \%$ of the phase-independent one, and therefore we neglect it. Thus, the collision-induced loss of the energy and number of atoms (for both solitons) are predicted by the perturbation theory to be

$$
(\triangle E)_{\mathrm{rad}}=\alpha \epsilon^{2} A^{7},(\triangle N)_{\mathrm{rad}}=(\alpha / 2) \epsilon^{2} A^{5},
$$

where $\alpha$ is the same numerical coefficient as in Eq. (15).

The energy of a free soliton and its norm (number of atoms), in the $\epsilon=0$ limit, are

$$
E_{\mathrm{sol}}=-\frac{1}{3} A^{3}+\frac{1}{2} M_{\mathrm{eff}} V^{2}, N_{\mathrm{sol}}=2 A
$$

(the negative term in $E_{\text {sol }}$ is the binding energy). First, the norm loss, $\triangle N$, taken from Eq. (18), gives rise to the collision-induced change of the soliton's amplitude: $\triangle A=-(\triangle N)_{\mathrm{rad}} / 2=-(\alpha / 4) \epsilon^{2} A^{5}$. The corresponding change in the binding (potential) energy of both solitons is positive,

$$
\triangle E_{\text {bind }} \equiv \triangle\left(-\frac{2}{3} A^{3}\right)=-2 A^{2} \triangle A=\frac{\alpha}{2} \epsilon^{2} A^{7} .
$$

Finally, the energy balance predicts a change in the total kinetic energy:

$$
\triangle E_{\mathrm{kin}}=-(\triangle E)_{\mathrm{rad}}-\triangle E_{\mathrm{bind}}=-(3 \alpha / 2) \epsilon^{2} A^{7} .
$$




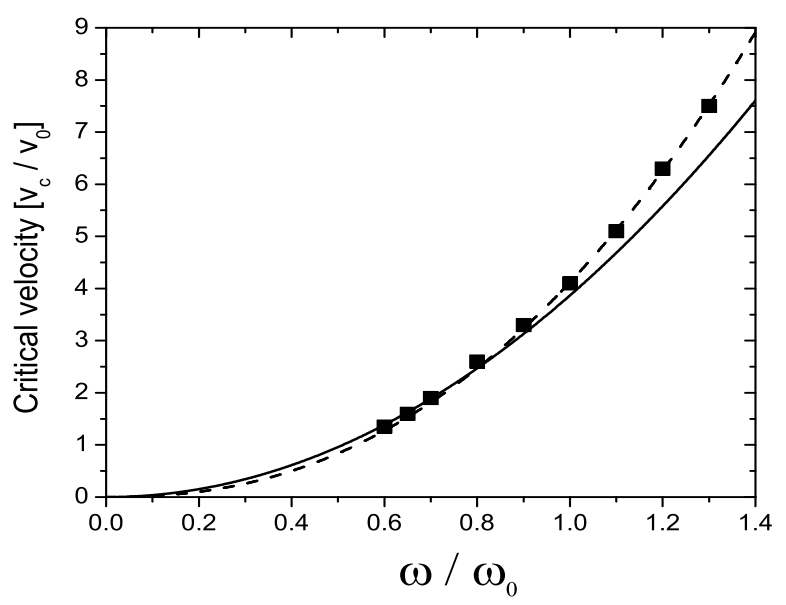

FIG. 2: The critical velocity for the merger of colliding solitons, $v_{c} / v_{0}$ (as an experimentally relevant reference value, we take $v_{0}=0.21 \mathrm{~mm} / \mathrm{s}$ ), as a function of the strength of the transverse confinement, $\omega / \omega_{0}$ (with $\omega_{0}=2 \pi \times 710 \mathrm{~Hz}$, as in Ref. [2]). For relatively weak confinement (smaller $\omega$ ), dependence $V_{c} \propto \omega^{2}$ is observed, as predicted by the perturbation theory (the solid line shows the $\omega^{2}$ power law as a guide to the eye). The dashed line is the power-law fit to the last four points of the numerical results, yielding $V_{c} \propto \omega^{2.29 \pm 0.07}$.

The merger condition states that the loss of the kinetic energy is equal to or exceeds the initial kinetic energy [21]. With regard to the expression for the total kinetic energy of both solitons which follows from Eq. (19), $E_{\text {kin }}=2 A V^{2}$, this condition means that the merger is expected if the initial velocity of each soliton falls below a critical value:

$$
V^{2}<V_{c}^{2}=\frac{3}{4} \alpha \epsilon^{2} A^{6} \equiv \frac{3 \alpha}{256} \epsilon^{2} N_{\mathrm{sol}}^{6} .
$$

The derivation of the merger threshold implies that the critical velocity is much smaller than the above-mentioned selfacquired velocity, $V_{\text {self }}=2 A$ (then, the initial velocities of the solitons may be disregarded in the above energy-balance analysis, in comparison with $V_{\text {self }}$, as it was actually done). Expression (22) indeed satisfies condition $V_{c} \ll V_{\text {self }}$, as $\epsilon$ is a small parameter.

\section{Numerical results}

For simulations of soliton collisions in Eq. (6), we chose parameter values close to those in the real experiment 2], where ${ }^{7} \mathrm{Li}$ atoms were used: a very small scattering length, $a=-0.06 \mathrm{~nm}$, transverse oscillation frequency $\omega=2 \pi \times 710 \mathrm{~Hz}$, and the number of atoms $N_{\text {sol }}=4000$. However, we did not include any external longitudinal potential, in contrast to the expulsive potential that was present in the experiment. Recall that the expulsive potential made the soliton stability region very small [2], and actually caused the soliton to be very close to the 3D limit. The present simulations do not include the external potential because we are interested not in effects produced by such a potential, but rather in small deviations from the one-dimensionality. In fact, a modification of the above-mentioned experimental setup, with the aim to make the central segment of the cigar-shaped trap free of any tangible axial potential, is quite possible.

To compare the analytical prediction for the critical velocity, given by Eq. (22), to numerical results, it is necessary to express perturbative parameter $\epsilon$ in terms of the transverse trapping frequency $\omega$. Undoing the above renormalizations, one arrives at a conclusion that Eq. (22) implies a quadratic dependence, $V_{c} \propto \omega^{2}$, within the framework of the perturbation theory. This dependence is indeed observed in simulations at relatively small $\omega$, as seen in Fig. (2). However, at larger $\omega$, i.e., for stronger transverse confinement, the numerical results feature a greater power in the $V_{c}(\omega)$ dependence. In particular, the best fit to the last four numerical points in Fig. (2) yields $V_{c} \propto \omega^{2.29 \pm 0.07}$, which demonstrates a small but tangible deviation from the power law corresponding to the perturbation limit.

We now turn to the dependence of the critical velocity on the number of atoms, $N$. The analytical prediction, Eq. (22), clearly implies $V_{c} \propto N^{3}$. In Fig. (3), this dependence is indeed observed at smaller values of $N$ (i.e., for weaker nonlinearity), where the perturbation limit should naturally be valid. Perusal of numerical data shows that, in this 


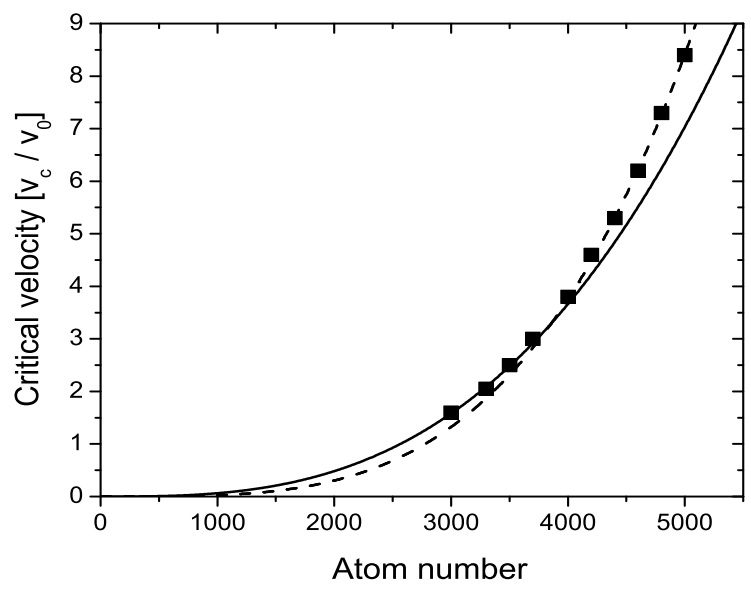

FIG. 3: The critical velocity for the merger of two solitons as a function of the number of atoms in each of the colliding solitons, $N_{\text {sol }}$. At smaller $N_{\text {sol }}$, i.e., for weaker nonlinearity, the $V_{c} \propto N_{\text {sol }}^{3}$ dependence is observed, as predicted by the perturbation theory, see Eq. (22). To say more accurately, the solid curve, which is the fit to the first four numerical points, features a power law $V_{c} \propto N_{\mathrm{sol}}^{2.9 \pm 0.2}$. The dashed curve is the power-law fit to the last four points, showing a different power dependence, $V_{c} \propto N_{\mathrm{sol}}^{3.62 \pm 0.05}$

range, the actual collision-induced radiation loss is very small, and, as a result, the merger does not lead to complete fusion of the colliding solitons into a single pulse, but rather to formation of a bound state of two solitons ("weak merger"), as can be seen in Fig. 4 Namely, after the first collision, the solitons re-emerge as two distinct wave packets which then collide again many times. A similar nearly radiationless inelastic collision, leading to the formation of a two-soliton loosely bound state, was recently observed in simulations of a weakly discrete cubic NLSE 22].

A definite deviation from the $V_{c} \propto N_{\text {sol }}^{3}$ dependence is observed in Fig. 3 for $N_{\text {sol }}>4000$, which shows a limitation of the perturbative predictions. In this regime of strong nonlinearity, a smooth transition in the collision process occurs, from the formation of the above-mentioned long-lived bound state to direct ("strong") merger of two solitons into a single pulse, which is accompanied by a burst of radiation. The conspicuous loss of matter with the radiation prevents the emerging single pulse from having the number of atoms above the collapse threshold, therefore the pulse does not blow up. The transition is expressed in reduction of the life time of the loose bound state before the complete merger. In Fig. [5 which represents the strongest nonlinearity included in the present framework, the bound state features only two oscillations.

For even stronger nonlinearities (which were also considered), the power-law dependence of $V_{c}$ on the number of atoms and transverse trapping frequency is observed in the form of $N_{\text {sol }}^{4}$ and $\omega^{3}$, respectively. However, in such an extreme regime, the relevance of the quasi-1D model is questionable. In any case, these results convey a clear caveat to soliton experiments, in which relative variations in the atom number may be as large as $\simeq 2$ : the strong power-law dependence of the critical velocity on the number of atoms should be taken into account, to avoid occasional merger of solitons.

\section{B. Symmetry breaking in soliton collisions with $\Delta \varphi \neq 0$}

We proceed to inelastic collisions of identical solitons with the phase difference of $0<\Delta \varphi<\pi / 2$. Numerical simulations of Eq. (6) show a salient effect of symmetry breaking in this case: while the solitons separate after the collision, they emerge as two pulses with different amplitudes (then, the velocities are also different, to comply with the momentum conservation), as shown in Fig. [6] It should be mentioned that a similar effect was observed in simulations of collisions between identical solitons in some other nonintegrable 1D models, chiefly in those describing transmission of nonlinear optical pulses, within the framework of the coupled-mode theory, in waveguides equipped with Bragg gratings. In that context, the collision-induced symmetry breaking was reported in basic single-core models [23], and in more sophisticated dual-core ones [24]. A similar effect was also observed in collisions between moving solitons in the discrete NLSE [25].

In order to achieve qualitative understanding of the symmetry breaking, we resort to consideration of an ansatz 


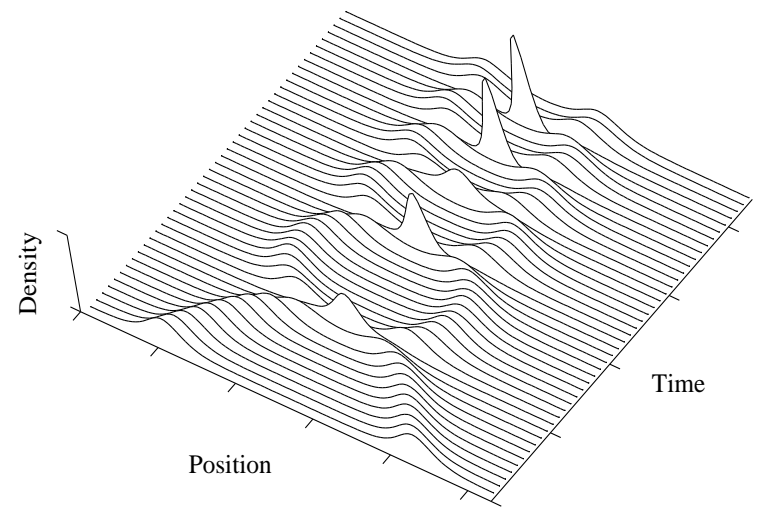

FIG. 4: Density profiles as a function of time in a regime of "weak" merger $\left(N_{\text {sol }}=3500, v / v_{0}=2\right)$. After the first collision, the two solitons re-appear as two distinct wave pulses which then collide again many times.

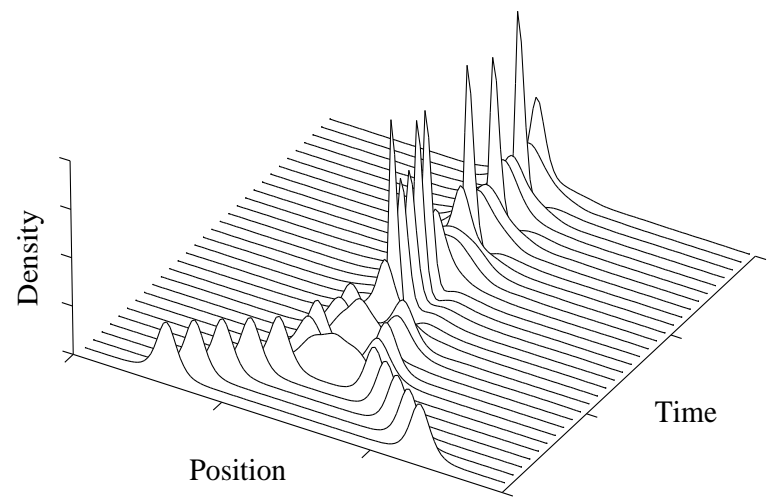

FIG. 5: The same as in Fig. 4 but in the regime of "strong" merger (for $N_{\text {sol }}=5000, v / v_{0}=8$ ). A short-lived bound state quickly merges into a single breather-like pulse.

based on a formal linear superposition of two completely overlapping identical solitons (7), at some moment of time $t=t_{0}$, with velocities $\pm V$ and phases $\pm(1 / 2) \triangle \varphi$. The ansatz yields the following expression:

$$
\Psi_{\text {ansatz }}(x, t)=2 e^{i\left(|\mu|-\frac{1}{2} V^{2}\right) t_{0}} \sqrt{\frac{|\mu|}{\sqrt{g^{2}+4|\mu|} \cosh (2 \sqrt{2|\mu|} x)+g}} \cos (V x+\triangle \varphi) .
$$

An essential peculiarity of this expression is that the central points of the two last multipliers do not coincide: one is found at $x=0$, while the other one at $x=-\Delta \varphi / V$. This simple observation suggests a concept of the mismatch between the amplitude center and phase center of the pair of colliding identical solitons. The mismatch was considered as a cause of breaking the symmetry between colliding solitons in the above-mentioned model based on the discrete NLSE 25]. 


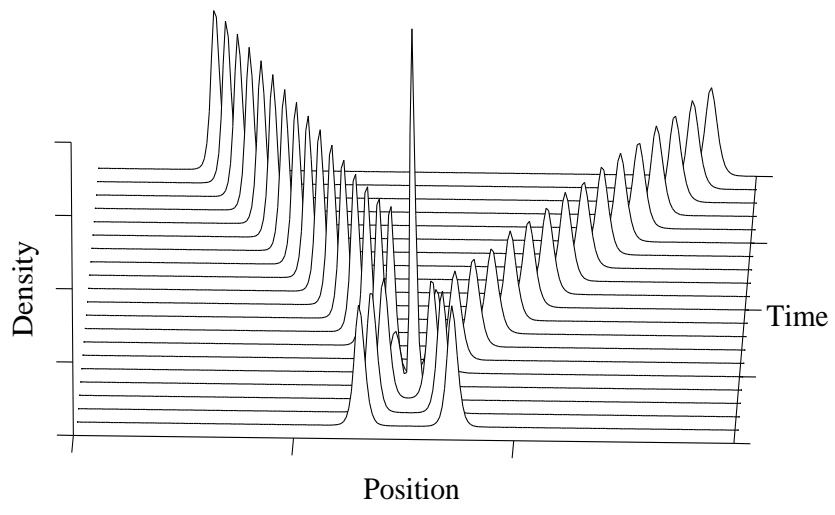

FIG. 6: Density profiles of solitons featuring the symmetry breaking in the collision, for $\Delta \varphi=\pi / 10$.

To characterize the asymmetry of ansatz (23) qualitatively, we introduce its center-of-mass coordinate,

$$
\xi(v) \equiv \frac{\int_{-\infty}^{+\infty} x\left|\phi_{\text {ansatz }}(x)\right|^{2} d x}{\int_{-\infty}^{+\infty}\left|\phi_{\text {ansatz }}(x)\right|^{2} d x}=\frac{\sin (2 \triangle \varphi)}{\sqrt{2|\mu|}} \frac{\gamma \cosh (\gamma v) \sinh (\pi v)-\pi \sinh (\gamma v) \cosh (\pi v)}{\sinh (\pi v)[\gamma \sinh (\pi v)+\pi \cos (2 \triangle \varphi) \sinh (\gamma v)]},
$$

with $v \equiv V / \sqrt{2|\mu|}$ and $\gamma \equiv \tan ^{-1}(2 \sqrt{|\mu|} / g)$. For the qualitative understanding of the situation, we adopt a natural conjecture that the strongest possible symmetry breaking is attained at a value of the velocity $v=v_{\max }$, which corresponds to a maximum of $|\xi|$ for given $\Delta \varphi$. For the weak quintic nonlinearity $\left(|\mu| \ll g^{2}\right)$, one has $\gamma \approx 2 \sqrt{|\mu|} / g$, and Eq. (24) simplifies:

$$
\xi(V) \approx \frac{\sin (2 \triangle \varphi)}{\sqrt{2|\mu|}} \frac{\sinh (\pi v)-\pi v \cosh (\pi v)}{\sinh (\pi v)[\sinh (\pi v)+\pi v \cos (2 \triangle \varphi)]},
$$

Asymmetry parameter $\xi$ is shown, as a function of $V$, in Fig. (7) by the solid line for $\triangle \varphi=\pi / 10$. It characterizes the degree of the collision-induced symmetry breaking, and predicts a maximum at some nonzero velocity. Quite a similar dependence is indeed produced by numerical simulations of Eq. (6) for the same value of $\triangle \varphi$, as shown by dots in Fig. (77). The dots display values of the amplitude ratio of the output soliton pair, as found from the simulations. Actually, the pulses emerging from the inelastic collisions are breathers with time-dependent amplitudes. Therefore, we averaged the amplitudes over long propagation distances after the collision.

Generally, the numerical data in Fig. (7) follow the predicted symmetry-breaking parameter. However, two notable deviations are observed: a nonsmooth shape of the numerical curve (local modulations near the maximum, which tend to give rise to two extra local maxima, definitely exceed an error of the numerical simulations), and a weakly decaying tail, which implies that the asymmetry generated by collisions between fast solitons is essentially larger than predicted by the above model. Note that the analytical model does not include radiation loss. The latter may enhance the asymmetry, as the loss itself is, plausibly, asymmetric too.

We note that Ref. 51, in which collisions of nearly 3D solitary waves were considered through simulations of the full 3D GPE, showed very little symmetry breaking ("population transfer") between colliding solitons with the initial phase difference of $\Delta \varphi=\pi / 10$, less than $1 \%$. However, our results predict that the matter transfer (symmetry breaking) would be conspicuous at specific values of the collision velocities, which might not be included in the analysis reported in Ref. [5]

For very small $\Delta \varphi$, we observed chaotic behavior in the output of the collision, similar to what was reported in a weakly discrete NLSE [22] (see also Ref. 26]). Very recently, chaotic behavior was predicted for collisions of more than two MW solitons, in the presence of a longitudinal parabolic trapping potential [27]. In our model, the collision between two solitons is sufficient to observe chaotic behavior, which will be reported elsewhere. 


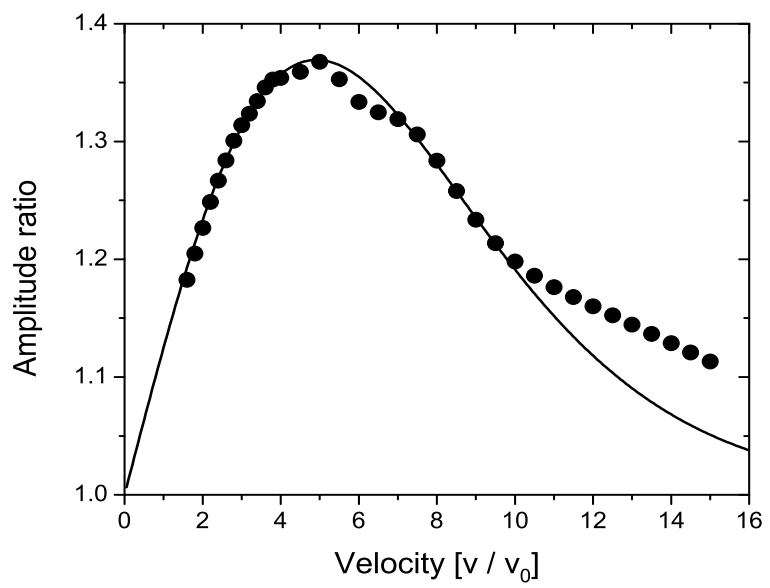

FIG. 7: Black circles show the numerically found amplitude ratio of two solitons after the symmetry-breaking collision for initial phase difference $\Delta \varphi=\pi / 10$. The solid line shows the velocity dependence of the symmetry-breaking parameter, as given by Eq. 25), for the same value of $\Delta \varphi$.

\section{CONCLUSIONS}

This work aims to understand how the tight confinement in transverse directions affects the longitudinal dynamics of matter-wave solitons in the quasi-1D setting. Within the framework of the known model, which reduces the multidimensional character of the full Gross-Pitaevskii equation to the appendage of an additional self-focusing quintic term to the effective 1D equation, we have investigated deviations from the ideal soliton behavior.

A family of exact stationary solutions for the solitons has been constructed, and it was demonstrated that the entire family is stable, despite the possibility of collapse in the modified $1 \mathrm{D}$ equation (with the negative scattering length). We have found inelastic effects in soliton collisions, which are impossible in ideal solitons. Two identical in-phase solitons merge into a single pulse, if the collision velocity is smaller than a critical value. In fact, two different types of the merger were observed, "strong" and "weak" ones, the former leading to the formation of a loose bound state of two solitons that feature repeated collisions, with very weak radiation loss, while the latter means direct fusion into a single pulse, which is accompanied by a burst of radiation (in that case, the radiation loss helps the emerging pulse to drop the number of atoms below the collapse threshold, and thus avoid the blowup). Both the analytical approximation, based on the perturbation theory, and numerical results highlight the strong dependence of the critical velocity on the strength of the transverse confinement and the number of atoms in the solitons. Symmetry breaking in collision between identical solitons with nonzero phase difference was also found, and partially explain by means of the calculation of a phenomenologically defined symmetry-breaking parameter, which measures the mismatch between amplitude and phase centers of the colliding solitons.

This work was supported, in a part, by the Israel Science Foundation, through grant No. 1125/04 (L.K.) and the Center-of-Excellence grant No. 8006/03 (B.A.M.).

[1] J. L. Roberts, N. R. Claussen, S. L. Cornish, E. A. Donley, E. A. Cornell, and C. E. Wieman, Phys. Rev. Lett. 86, 4211 (2001); J. M. Gerton, D. Strekalov, I. Prodan, and R. G. Hulet, Nature 408, 692 (2000); E. A. Donley, N. R. Claussen, S. L. Cornish, J. L. Roberts, E. A. Cornell, and C. E. Wieman, ibid. 412, 295 (2001).

[2] L. Khaykovich, F. Schreck, G. Ferrari, T. Bourdel, J. Cubizolles, L. D. Carr, Y. Castin, and C. Salomon, Science 256, 1290 (2002).

[3] K. E. Strecker, G. B. Partridge, A. G. Truscott and R. G. Hulet, Nature 417, 150 (2002).

[4] S. Sinha, A. Y. Cherny, D. Kovrizhin, and J. Brand, Phys. Rev. Lett. 96, 030406 (2006).

[5] N. G. Parker, A. M. Martin, S. L. Cornish and C. S. Adams, cond-mat/0603059 (2006).

[6] B. B. Baizakov, B. A. Malomed and M. Salerno, Phys. Rev. A 70, 053613 (2004), and an article in Nonlinear Waves: Classical and Quantum Aspects, ed. by F. Kh. Abdullaev and V. V. Konotop, pp. 61-80 (Kluwer Academic Publishers: Dordrecht, 2004; also available at http://rsphy2.anu.edu.au/〜 asd124/Baizakov_2004_61_NonlinearWaves.pdf). 
[7] S. L. Cornish, S. T. Thompson and C. E. Wieman, Phys. Rev. Lett. 96, 170401 (2006).

[8] A. E. Muryshev, G. V. Shlyapnikov, W. Ertmer, K. Sengstock, and M. Lewenstein, Phys. Rev. Lett. 89, 110401 (2002).

[9] V. M. Pérez-García, H. Michinel, and H. Herrero, Phys. Rev. A 57, 3837 (1998).

[10] M. I. Weinstein, Commun. Math. Phys. 87, 567 (1983); L. Bergé, T. J. Alexander, and Y. S. Kivshar, Phys. Rev. A 62, 023607 (2000).

[11] L. D. Carr, and J. Brand, Phys. Rev. Lett 92, 040401 (2004); Phys. Rev. A 70, 033607 (2004).

[12] L. Salasnich, A. Parola, and L. Reatto, Phys. Rev. A 65, 043614 (2002).

[13] A. Gammal, L. Tomio, and T. Frederico, Phys. Rev. A 66, 043619 (2002).

[14] Y. B. Band, I. Towers, and B. A. Malomed, Phys. Rev. A 67, 023602 (2003).

[15] L. D. Carr and Y. Castin, Phys. Rev. A 66, 063602 (2002).

[16] Kh. I. Pushkarov, D. I. Pushkarov, and I. V. Tomov, Opt. Quant. Electr. 11, 471 (1979); S. Cowan, R. H. Enns, S. S. Rangnekar, and S. S. Sanghera Can. J. Phys. 64, 311 (1986).

[17] Yu. Kagan, A. E. Muryshev, and G. V. Shlyapnikov, Phys. Rev. Lett. 81, 933 (1998); F. Kh. Abdullaev, A. Gammal, L. Tomio, and T. Frederico, Phys. Rev. A 63, 043604 (2001).

[18] M. G. Vakhitov and A. A. Kolokolov, Izv. Vuz. Radiofiz. 16, 1020 (1973) [in Russian; English translation: Sov. J. Radiophys. Quantum Electr. 16, 783 (1973)].

[19] R. W. Micallef, V. V. Afanasjev, Y. S. Kivshar, and J. D. Love, Phys. Rev. E 54, 2936 (1996).

[20] V. I. Karpman and V. V. Solov'ev, Physica D 3, 487 (1981).

[21] Y. S. Kivshar and B. A. Malomed, Rev. Mod. Phys. 61, 763 (1989).

[22] S. V. Dmitriev and T. Shigenari, Chaos 12, 324 (2002).

[23] W. C. K. Mak, B. A. Malomed, and P. L. Chu, Phys. Rev. E 68, 026609 (2003); D. R. Neill and J. Atai, Phys. Lett. A 353, 416 (2006).

[24] J. Atai and B. A. Malomed, Phys. Rev. E 64, 066617 (2001); Phys. Lett. A 298, 140 (2002); ibid. 342, 404 (2005).

[25] I. E. Papacharalampous, P. G. Kevrekidis, B. A. Malomed and D. J. Frantzeskakis, Phys. Rev. E 68, 046604 (2003).

[26] S. V. Dmitriev, P. G. Kevrekidis, B. A. Malomed, and D. J. Frantzeskakis, Phys. Rev. E 68, 056603 (2003).

[27] A. D. Martin, C. S. Adams, and S. A. Gardiner, cond-mat/0604086 (2006). 